\title{
RETRACTED ARTICLE: Molecular diagnosis of leishmaniasis at the subgenus and species level
}

\author{
Krishna Khairnar $^{1}$ - Carrie Lynde ${ }^{2}$ - Filip Ralevski ${ }^{3}$. \\ Jay Keystone ${ }^{2} \cdot$ Anne McCarthy $^{4} \cdot$ Jeremy Levine $^{4}$. \\ Dylan R. Pillai ${ }^{5}$
}

Received: 14 August 2013 / Accepted: 18 October 2013

(C) Springer-Verlag Berlin Heidelberg 2013

The article "Molecular diagnosis of leishmaniasis at the subgenus and species level" published online 5 November 2013 in Parasitology Research, DOI 10.1007/s00436-013-3663-1, has been retracted by the publisher and the journal Editors Heinz Mehlhorn and Bill Chobotar. The retraction has been agreed to due to the fact that although all the data reported in the figures of the main manuscript and in the supporting information are correct, incorrect clinical diagnoses could be made from the way the data are interpreted currently in the paper. Cross testing a newly designed assay against positive clinical samples alone is insufficient evidence of its specificity. This conclusion can be traced back to a misinterpretation of experimental data in the main manuscript, for which the authors accept full responsibility. The online version of this article contains the full text of the retracted article as electronic supplementary material.
Electronic supplementary material The online version of this article (doi:10.1007/s00436-013-3663-1) contains supplementary material, which is available to authorized users.

Krishna Khairnar

kskhairnar@gmail.com

1 Council for Scientific and Industrial Research, National Environmental Engineering and Research Institute (CSIR-NEERI), Nagpur 440020, India

2 Department of Medicine, University of Toronto, Toronto, ON, Canada

3 Public Health Laboratory, OAHPP, Toronto, ON, Canada

4 Infectious Diseases Division, The Ottawa Hospital, Ottawa, ON, Canada

5 Department of Pathology \& Laboratory Medicine, University of Calgary, Calgary, AB, Canada 